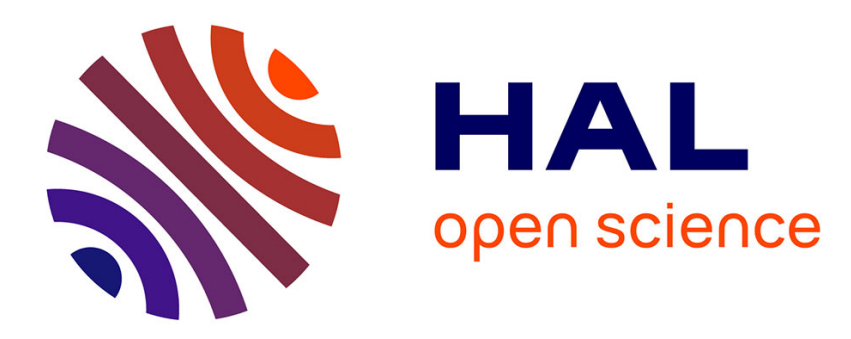

\title{
Comparison of the Lambert W-function based solutions to the Colebrook equation
}

Dejan Brkić

\section{To cite this version:}

Dejan Brkić. Comparison of the Lambert W-function based solutions to the Colebrook equation. Engineering Computations, 2012, 29 (6), pp.617-630. 10.1108/02644401211246337 . hal-01586546

\section{HAL Id: hal-01586546 https://hal.science/hal-01586546}

Submitted on 18 Sep 2017

HAL is a multi-disciplinary open access archive for the deposit and dissemination of scientific research documents, whether they are published or not. The documents may come from teaching and research institutions in France or abroad, or from public or private research centers.
L'archive ouverte pluridisciplinaire HAL, est destinée au dépôt et à la diffusion de documents scientifiques de niveau recherche, publiés ou non, émanant des établissements d'enseignement et de recherche français ou étrangers, des laboratoires publics ou privés. 


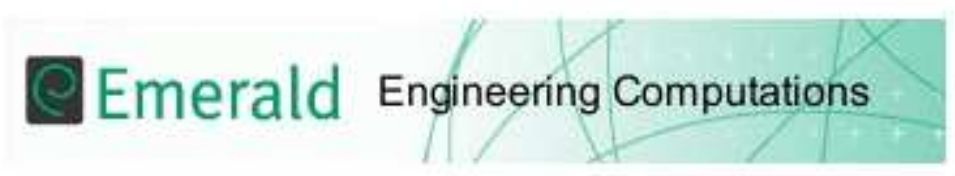

\section{Comparison of the Lambert W-Function Based Solutions to the Colebrook Equation}

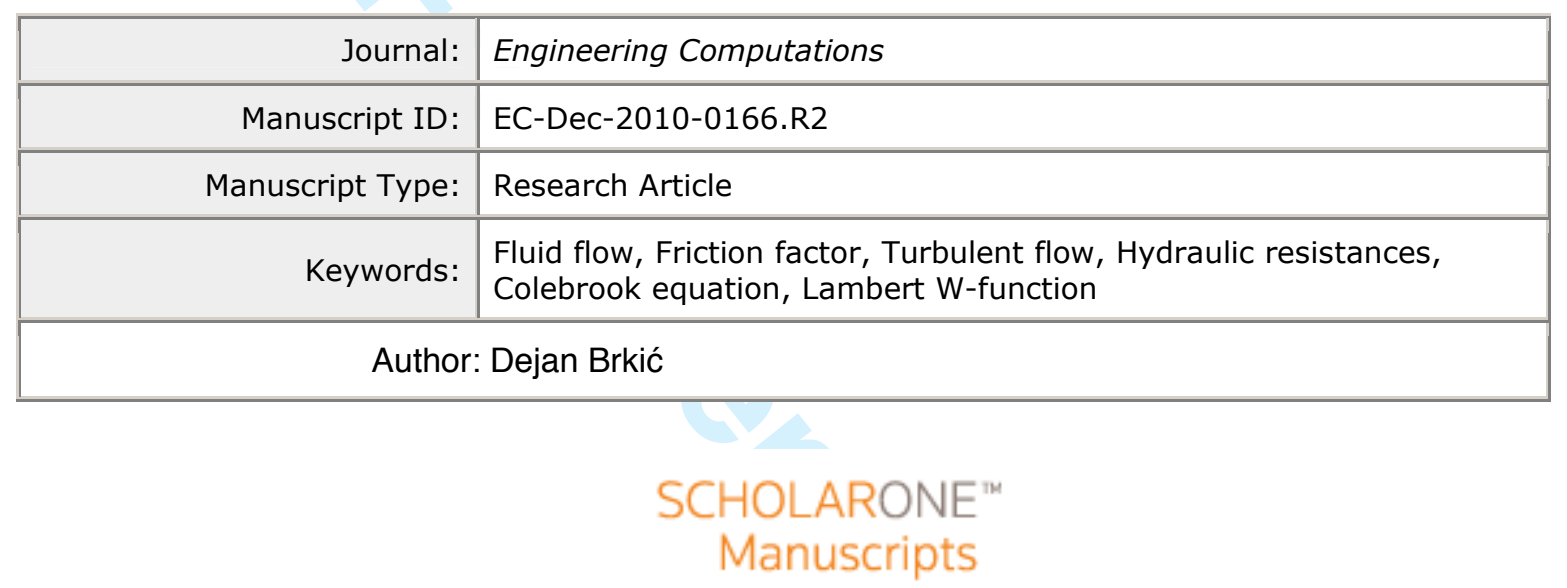




\section{Comparison of the Lambert W-Function Based Solutions to the Colebrook Equation}

\section{Structured abstract:}

Purpose: The Colebrook equation for determination of hydraulic resistances is implicit in fluid flow friction factor and hence it has to be approximately solved using iterative procedure or using some of the approximate explicit formulas developed by many authors.

Design/methodology/approach: Alternate mathematically equivalents to the implicit Colebrook equation in explicit form with no approximation involved actually exist.

Findings: These alternate equations were developed using Lambert W-function. The paper compares various implementations of the Lambert W methodology and shows that some of these are less able than others to yield solutions using modern computer hardware. This is because the functions require the evaluation of terms with numerical values outside the ranges that can be expressed on most computers.

Research limitations/implications: Some of existed transformations cannot be applied for high values of relative roughness of inner pipe surface and the Reynolds number. This limitation applied only for computer computations. Other presented transformations do not sufferer of this limitation.

Practical implications: Presented procedures can be easily implemented in a computer code. Recommended solution can be used in all cases that can occur in engineering practice. Originality/value: Here will be shown some possible practical procedures for solution to the transformed Colebrook equation. Accuracy analysis and comparisons of presented formulas are also performed. Recommendation for use is shown. 
Keywords: Fluid flow; Friction factor; Turbulent flow; Hydraulic resistances; Colebrook equation; Lambert W-function

\section{Introduction}

The empirical Colebrook equation is valid for turbulent regime in smooth and rough pipes with special attention on transitional regime between them (1).

$$
\frac{1}{\sqrt{\lambda}}=-2 \cdot \log \left(\frac{2.51}{\operatorname{Re} \cdot \sqrt{\lambda}}+\frac{\varepsilon}{3.71 \cdot D}\right)
$$

The Colebrook equation has two parts, i.e. turbulent smooth and turbulent rough part which can be noted separately. The Colebrook equation unites these two parts in one coherent equation but also Colebrook was not attempting to find some sort of approximation to a sum of the smooth and rough expressions. Actually, Colebrook was seeking a combined equation that is asymptotic to the smooth and rough expressions. This new coherent equation covers transitional zone between smooth and rough regime. First addend in bracket of previous equation is implicitly given and it represents smooth regime (2).

$$
\frac{1}{\sqrt{\lambda}}=-2 \cdot \log \left(\frac{2.51}{\operatorname{Re} \cdot \sqrt{\lambda}}\right)=2 \cdot \log (\operatorname{Re} \cdot \sqrt{\lambda})-0.8
$$

Similarly the rough portion of turbulence is represented by second addend of the Colebrook equation (3).

$$
\frac{1}{\sqrt{\lambda}}=-2 \cdot \log \left(\frac{\varepsilon}{3.71 \cdot D}\right)=1.74-2 \cdot \log \left(\frac{2 \cdot \varepsilon}{D}\right)=1.14-2 \cdot \log \left(\frac{\varepsilon}{D}\right)
$$

Mathematically, shown unification is incorrect i.e. $\log (A+B) \neq \log (A)+\log (B)$, but physically this approach gives good results. Problem can be treated as inverse; according to the logarithmic 
rules equally is incorrect to split the Colebrook relation into two pieces. How well Colebrook's equation smoothing contact between the turbulent smooth and the turbulent rough regime can be best seen in the graphical interpretation (Figure 1).

Figure 1. Colebrook relation make transitional curve between hydraulically smooth regime and turbulent rough regime

Today, the Colebrook equation for calculation of hydraulic flow friction factor is almost worldwide accepted as a standard. It was developed in 1930's by Colebrook (1939) who used data from the experiment conducted by his colleague, Professor White and himself (Colebrook and White 1937). Later in 1940's, Rouse and Moody used Colebrook's equation for their famous charts (Moody 1944). Since then, the Colebrook equation has been historically adopted as sufficiently accurate for engineering applications although it is often incorrect. Even Moody (1944) stated the accuracy of his diagram is about $\pm 5 \%$ for smooth pipes and $\pm 10 \%$ for rough pipes. In spite of that, the Colebrook equation is replaced very slowly by new equations such as those developed using the data from the Princeton super-pipe or the Oregon facility (Cordero 2008). Actually, many possible equations can be found that are asymptotic to the smooth and rough expressions. Colebrook chose one and compared it with limited experimental data available to him. Since different types of roughness cause different forms of transition from rough to smooth, it is clear that the Colebrook equation should not be regarded as special. It was the first and that it is now widely used even though hardly anybody seems to acknowledge its considerable limitations. 
Alternate mathematically equivalents to the original expression of the Colebrook equation in explicit form with no approximation involved actually exist (More 2006, Sonnad and Goudar 2007, Clamond 2009, Brkić 2011a). These alternate equations were developed using the Lambert W-function. Some of the existed transformations cannot be applied for high values of relative roughness of inner pipe surface and the Reynolds number (Sonnad and Goudar 2004). This limitation is applied only for computer computations. Other presented transformations do not sufferer of such limitation as will be shown in this paper.

\section{Derivate of explicit Lambert W-function based Colebrook equation}

The Lambert W-function is define by relation $\mathrm{W}(\mathrm{x}) \cdot \mathrm{e}^{\mathrm{W}(\mathrm{x})}=\mathrm{x}$, where $\mathrm{W}$ is the Lambert function and $\mathrm{x}$ is its argument (Corless et al 1996, Hayes 2005). For the real values of the argument $\mathrm{x}$, the $\mathrm{W}$-function has two branches, $\mathrm{W}_{0}$ (the principal branch) and $\mathrm{W}_{-1}$ (the negative branch). Only upper branch $\mathrm{W}_{0}$ is used for the transformations of Colebrook's equation in explicit form (Figure 2).

Figure 2. Detailed view of the Lambert W-function

Goudar and Sonnad (2003) show the explicit friction factor correlation for turbulent flow in smooth pipes which was developed using the Lambert W-function and the smooth part of the Colebrook equation. Using this approach (Brkić 2011a), Colebrook's equation can be also expressed in the explicit form where some transformation of its smooth part should be performed as follows (4). 


$$
\begin{aligned}
& \frac{1}{\sqrt{\lambda}}=-2 \cdot \log \left(\frac{2.51}{\operatorname{Re} \cdot \sqrt{\lambda}}\right)=-a \cdot \ln \left(\frac{2.51}{\operatorname{Re} \cdot \sqrt{\lambda}}\right)=-a \cdot \ln \left(\frac{2.51}{\operatorname{Re}}\right)-a \cdot \ln \left(\frac{1}{\sqrt{\lambda}}\right) \\
& \left.\begin{array}{l}
a=\frac{2}{\ln (10)} \Rightarrow \Phi=\frac{1}{a \cdot \sqrt{\lambda}} \Rightarrow a \cdot \Phi+a \cdot \ln (a \cdot \Phi)=a \cdot \ln \left(\frac{\operatorname{Re}}{2.51}\right) \Rightarrow \Phi+\ln (\Phi)=\ln \left(\frac{\operatorname{Re}}{2.51 \cdot a}\right) \\
W(x)+\ln [W(x)]=\ln (x) \Rightarrow \Phi=W\left[\frac{\operatorname{Re}}{2.51 \cdot a}\right] \Rightarrow \frac{1}{\sqrt{\lambda}}=a \cdot W\left[\frac{\operatorname{Re}}{2.51 \cdot a}\right] \Rightarrow \frac{1}{\sqrt{\lambda}}=\frac{2}{\ln (10)} W\left[\frac{\operatorname{Re} \cdot \ln (10)}{2 \cdot 2.51}\right] \\
\frac{1}{\sqrt{\lambda}}=2 \cdot \log \left(\frac{\operatorname{Re} \cdot \sqrt{\lambda}}{2.51}\right)=-2 \cdot \log \left(10^{\frac{-1}{\ln (10)} W\left[\frac{\operatorname{Re} \cdot \ln (10)}{5 \cdot 02}\right]}\right) \Rightarrow \frac{\operatorname{Re} \cdot \ln (10)}{2 \cdot 2.51}=10^{-2 \cdot \log \left(\frac{2.51}{\operatorname{Re} \cdot \frac{1}{\sqrt{\lambda}}}\right)}
\end{array}\right\}
\end{aligned}
$$

After that implicit expression in the Colebrook equation can be replaced with the Lambert Wfunction while its second so called "rough" addend remains untouched (5).

$$
\left.\begin{array}{l}
\frac{1}{\sqrt{\lambda}}=-2 \cdot \log \left(\frac{5.02 \cdot W\left[\frac{\mathrm{Re} \cdot \ln (10)}{5.02}\right]}{\operatorname{Re} \cdot \ln (10)}+\frac{\varepsilon}{3.71 \cdot D}\right) \\
x=\frac{\mathrm{Re} \cdot \ln (10)}{5.02}
\end{array}\right\}
$$

Set of equations (5) was developed using the original idea of Colebrook to bridge the gap smoothing the line between hydraulically "smooth" turbulent flow and turbulent flow in rough pipes as shown before in figure 1. No constraints in ranges for values of the Reynolds number and relative roughness in comparisons to the original Colebrook relation do not exist (as will be shown in this paper). But, beside presented reformulations of the Colebrook equation, in the papers of More (2006), Sonnad and Goudar (2007) and Nandakumar (2007), another possible transformation of the Colebrook equation using the Lambert-W function is shown. But relation (6) shown in these papers has limitation in applicability for high values of the Reynolds number and relative roughness as shown in Sonnad and Goudar (2004) because today available computers cannot operate with the extremely large numbers. 


$$
\left.\begin{array}{l}
\frac{1}{\sqrt{\lambda}}=\frac{2}{\ln (10)} \cdot W\left[x \cdot e^{x \cdot \frac{\varepsilon}{3.71 \cdot D}}\right]-\frac{\varepsilon}{3.71 \cdot D} \cdot \frac{\operatorname{Re}}{2.51} \\
x=\frac{\operatorname{Re} \cdot \ln (10)}{5.02} \\
x_{1}=x \cdot e^{x \cdot \frac{\varepsilon}{3.71 \cdot D}}
\end{array}\right\}
$$

In previous equation (6) argument of the Lambert W-function has changed. It is now noted as $\mathrm{x}_{1}$ where this new argument contains the old argument $\mathrm{x}$ from the set of equations (5). However, the numerical solution of eq. (6) using computers is not possible for all values of relative roughness and the Reynolds number and their combinations which can be occurred in engineering practice. Problem is in the exponential term in the argument $\mathrm{x}_{1}$ of the $\mathrm{W}$-function which increases rapidly. In fact, infinite combinations of relative roughness and the Reynolds number values exist for which the argument $\mathrm{x}_{1}$ of the $\mathrm{W}$-function becomes too large even for today very powerful computers. Argument of the Lambert W-function has to be smaller than $1.79 \cdot 10^{308}$ because registers in memory of computers have limited capacity (Sonnad and Goudar 2004).

\section{Solutions of alternate equations, accuracy requirements and error distribution}

The main aim of this paper is not to investigate accuracy or computational burden (i.e. speed) of presented procedures for solution of the Lambert W based Colebrook equation. Accuracy of alternate representations of the Colebrook equation depends only on accuracy of used approximate solution of the Lambert $\mathrm{W}$-function. Besides the relative simplicity of an explicit form, presented equations allow highly accurate estimation of friction factor as the Lambert Wfunction can be evaluated accurately. But, the main purpose of this paper is to compare different Lambert $\mathrm{W}$ based solutions of the Colebrook equation and to make comparisons among them. Or 
better to say, main purpose is to identify some constraints in applicability of certain solutions whereas other solutions do not suffer of such limitations.

Clamond (2009) provides MATLAB and FORTRAN codes for the Colebrook relation expressed in term of the Lambert W-function. Keady (1998) also provides Maple interpretation of here presented problem.

Since the Lambert $\mathrm{W}$ is transcendental function, its formal solution can be expressed only in endless form (7). Similar as using iterative solution, accuracy of formal solution depends on number of terms used to form "tower" in eq. (7).

$$
\mathrm{W}(\mathrm{x})=\ln \frac{\mathrm{x}}{\ln \left(\frac{\mathrm{x}}{\ln \left(\frac{\mathrm{x}}{\ln (\cdot)}\right)}\right)}
$$

After Boyd (1998), instead $\mathrm{W}=\mathrm{W}(\mathrm{x})$ it is convenient to define a new function $\omega=\omega(\mathrm{y})$ as auxiliary tool (8).

$$
\left.\begin{array}{c}
\omega=W+1 \\
y=1+x \cdot e^{1} \approx 1+2.71 \cdot \mathrm{x} \\
\xi=\frac{\sqrt{2 \cdot y}}{\ln (10)-\ln (\ln (10))} \\
\tanh (\xi)=\left(e^{\xi}-e^{-\xi}\right) /\left(e^{\xi}+e^{-\xi}\right) \\
\omega_{0}=\{\ln (y+10)-\ln (\ln (y+10))\} \cdot \tanh (\xi) \\
\Omega=\frac{\left(\ln (y)-\frac{7}{5}\right) \cdot e^{-\frac{3}{40} \cdot\left(\ln (y)-\frac{7}{5}\right)^{2}}}{10} \\
\omega_{i+1}=\omega_{i}-\frac{\left(\omega_{i}-1\right)-e^{-\omega_{i} \cdot(y-1)}}{\omega_{i}}
\end{array}\right\}
$$


Barry et al (2000) proposed approximate solution for the upper branch of the Lambert W-

function which is valid for solution of here presented problem (9). Also, improved version eq. (9) can be seen in Barry et al (2000).

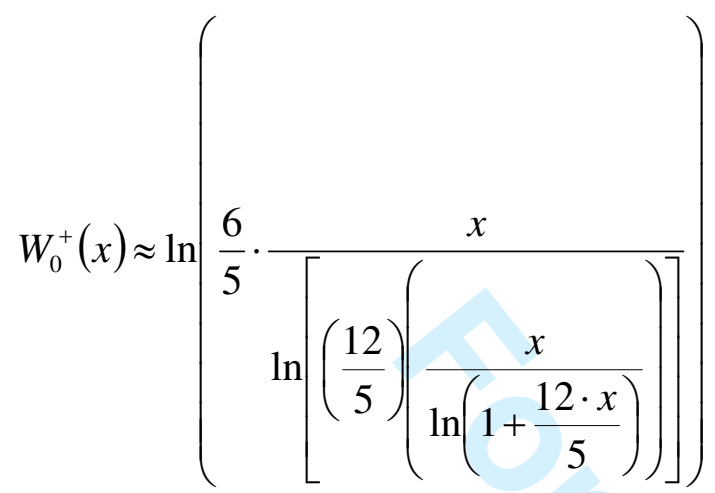

Winitzki (2003) also proposed global approximation to the Lambert W-function (10).

$\mathrm{W}(\mathrm{x}) \approx \ln (1+\mathrm{x}) \cdot\left(1-\frac{\ln (1+\ln (1+\mathrm{x}))}{2+\ln (1+\mathrm{x})}\right)$

Two numerical examples are solved in tables 1 and 2 using formulas shown in this section of the text. These two examples are marked also in figure 1 and 3 to 9 . The implicit Colebrook equation today can be easily solved using some kind of spreadsheet solvers (iterative solution). Here will be used as standard, solution calculated using MS Excel ver. 2007. Since iterative solution after enough number of iterations can be accepted as accurate, it will be used for comparisons in examples 1 and 2. Approach with set of equations (5) will be used in table 1 while approach with set of equations (6) will be used in table 2.

Table 1. Solutions of the explicit, Lambert W-function based Colebrook equation (5)

Limitation of formulas presented by set of equations (6) for high values of the Reynolds number and relative roughness is shown in table 2. 
Table 2. Solutions of the explicit, Lambert W-function based Colebrook equation (6)

\begin{abstract}
Main difference between the calculation in table 1 and table 2 is in the argument of the Lambert $\mathrm{W}$-function $\mathrm{x}$ and $\mathrm{x}_{1}$, respectively. Argument $\mathrm{x}$ is never too large for computer register while in other approach, argument $\mathrm{x}_{1}$ is. Of course, argument $\mathrm{x}$ is not too large only if the Reynolds number and relative roughness are in the range which occurs in engineering practice.
\end{abstract}

Error distribution for solution of equations (5) and (6) using procedure by Boyd (1998) is shown in figures 3 and 4, respectively. Similar for the procedure proposed by Barry et al (2000) and Winitzki (2003) error distribution can be seen in figures 5 and 6, and 7 and 8 .

Figure 3. Distribution of error of solution of explicit Lambert W-function based Colebrook equation (5) by Boyd (1998)

Figure 4. Distribution of error of solution of explicit Lambert W-function based Colebrook equation (6) by Boyd (1998)

Figure 5. Distribution of error of solution of explicit Lambert W-function based Colebrook equation (5) by Barry et al (2000)

Figure 6. Distribution of error of solution of explicit Lambert W-function based Colebrook equation (6) by Barry et al (2000)

Figure 7. Distribution of error of solution of explicit Lambert W-function based Colebrook equation (5) by Winitzki (2003) 
Figure 8. Distribution of error of solution of explicit Lambert W-function based Colebrook equation (6) by Winitzki (2003)

The distribution of error shown in figures 3, 5 and 7 are for the set of equations (5). Distribution of error is sensitive and therefore similar set of equations (11) produce different distribution of error (Figure 9) but calculation is still possible for all practical range of the Reynolds number and relative roughness.

$$
\left.\begin{array}{l}
\frac{1}{\sqrt{\lambda}}=-2 \cdot \log \left(10^{\frac{-1}{\ln (10)} W\left[\frac{\mathrm{Re} \cdot \ln (10)}{5.02}\right]}+\frac{\varepsilon}{3.71 \cdot D}\right) \\
x=\frac{\operatorname{Re} \cdot \ln (10)}{5.02}
\end{array}\right\}
$$

Figure 9. Distribution of error of solution of explicit Lambert W-function based Colebrook equation (11) by Barry et al (2000)

\section{Conclusion}

The Colebrook equation can be expressed in explicit form using the Lambert W-function. Related approximate solutions of the Lambert W-function are shown in this paper. Accuracy of some available Lambert W based forms of the Colebrook equation from literature is investigated (More 2006, Sonnad and Goudar 2007, Nandakumar 2007, Brkić 2011a). Also, related approximate solutions of the Lambert W-function are shown. Some already known limitation in use of some of available formulas is confirmed (Sonnad and Goudar 2004). Other available formulas do not suffer of these limitations for practical use (Brkić 2011a). The maximal error of these new formulas compared with iterative solution of Colebrook equation is up to $2 \%$ or 
slightly above (Figures 3, 5 and 7). Error distribution of new formulas (set of equations 5 and 11) is stabile or better to say, distribution of error does not change with change of procedure for solution of the Lambert W-function. Transformation of the Colebrook equation based on the Lambert W-function by More (2006), Sonnad and Goudar (2007), Nandakumar (2007) cannot be used for all practical combination of the Reynolds number and relative roughness (Figures 4, 6, 8). Reason is that typical IEEE-compliant machines cannot operate with numbers larger than about $10^{308}$ (Figure 10).

Figure 10. Constraints for using of a possible Lambert W function-based explicit Colebrook equation

Review of available explicit approximations to the Colebrook equation can be seen in Brkić (2011b). It has to be said that use of an explicit approximation do not necessary mean that the computational burden is reduced in comparison to iterative approach (Giustolisi et al 2011). Actually, main purpose of this paper is to compare the different formulations of Lambert W based Colebrook equation available in literature (More 2006, Sonnad and Goudar 2007, Nandakumar 2007, Brkić 2011a). The paper compares various implementations of the Lambert W methodology and shows that some of these are less able than others to yield solutions using modern computer hardware. This is because the functions require the evaluation of terms with numerical values outside the ranges that can be expressed on most computers. Approach by Brkić (2011a) does not suffer of that constraints and hence it can be recommended since it can be use for the whole range of the Reynolds number and relative roughness that can be occured in engineering practice. Approach by More (2006), Sonnad and Goudar (2007) and Nandakumar 
(2007) suffers of certain limitations as it is explained in this paper as well as in Sonnad and Goudar (2004). Decision whether someone will use the Lambert W based solution proposed by Brkić (2011a), iterative solution or some of explicit approximate formulas presented in Brkić (2011b), requires deeper analysis.

\author{
Nomenclature \\ $\mathrm{x}, \mathrm{x}_{1}$-arguments of the Lambert $\mathrm{W}$-function \\ W-in this text referred to the positive branch of Lambert function \\ $\omega$-shifted, auxiliary function proposed by Boyd (1998) \\ $y$ - argument of the shifted, auxiliary function $\omega$ \\ D-inner pipe diameter $(\mathrm{m})$ \\ Re-Reynolds number (dimensionless) \\ $\varepsilon$-roughness of the inner surface of pipe (m) \\ ع/D-relative roughness of the inner surface of pipe (dimensionless) \\ $\lambda$-Darcy, Darcy-Weisbach or Moody friction factor (dimensionless) \\ a, $\xi, \Phi, \Omega$-auxiliary term defined in the text \\ i-counter
}

\title{
References
}

Barry, D.A., Parlange, J.Y., Li, L., Prommer, H., Cunningham, C.J. and Stagnitti, F. (2000), “Analytical approximations for real values of the Lambert W-function”, Mathematics and Computers in Simulation, Vol. 53 No. 1-2, pp. 95-103. 
Boyd, J.P. (1998), “Global approximations to the principal real-valued branch of the Lambert Wfunction", Applied Mathematics Letters, Vol. 11 No. 6, pp. 27-31.

Brkić, D. (2011a), "W solutions of the CW equation for flow friction", Applied Mathematics Letters, Vol. 24 No. 8, pp. 1379-1383.

Brkić, D. (2011b), "Review of explicit approximations to the Colebrook relation for flow friction”, Journal of Petroleum Science and Engineering, Vol. 77 No. 1, pp. 34-48.

Clamond, D. (2009), "Efficient resolution of the Colebrook equation", Industrial and Engineering Chemistry Research, Vol. 48 No. 7, pp. 3665-3671.

Colebrook, C.F. (1939), “Turbulent flow in pipes with particular reference to the transition region between the smooth and rough pipe laws", Journal of the Institution of Civil Engineers. (London), Vol. 11 No 4, pp. 133-156.

Colebrook, C.F. and White, C.M. (1937), "Experiments with fluid friction in roughened pipes", Proceedings of the Royal Society of London. Series A, Mathematical and Physical Sciences, Vol. 161, No. 906, pp. 367-381.

Cordero, G.O. (2008), “An improved experimental correlation for Darcy friction factor”, Hydrocarbon Processing, Vol. 87 No. 7, pp. 97-99.

Corless, R.M., Gonnet, G.H., Hare, D.E.G., Jeffrey, D.J. and Knuth, D.E. (1996), “On the Lambert W function", Advanced in Computational Mathematics, Vol. 5 No. 1, pp. 329-359.

Goudar, C.T. and Sonnad, J.R. (2003), "Explicit friction factor correlation for turbulent flow in smooth pipes", Industrial and Engineering Chemistry Research, Vol. 42 No. 12, pp. 2878-2880.

Giustolisi, O., Berardi, L. and Walski, T.M. (2011), "Some explicit formulations of ColebrookWhite friction factor considering accuracy vs. computational speed", Journal of Hydroinformatics, Vol. 13 No. 3, pp. 401-418. 
Hayes, B. (2005), “Why W?”, American Scientist, Vol. 93, No. 2, pp. 104-108.

Keady, G. (1998), “Colebrook-White formulas for pipe flow”, Journal of Hydraulic Engineering ASCE, Vol. 124 No. 1, pp. 96-97.

Moody, L.F. (1944), "Friction factors for pipe flow", Transactions of the ASME, Vol. 66 No. 8, pp. 671-684.

More, A.A. (2006), “Analytical solutions for the Colebrook and White equation and for pressure drop in ideal gas flow in pipes”, Chemical Engineering Science, Vol. 61 No. 16, pp. 5515-5519. Nandakumar, V. (2007), “Analytical calculation of fanning friction factor”, Hydrocarbon Processing, Vol. 86 No. 1, pp. 97-100.

Sonnad, J.R. and Goudar, C.T. (2004), “Constraints for using Lambert W Function-based explicit Colebrook-White equation", Journal of Hydraulic Engineering ASCE, Vol. 130 No. 9, pp. 929-931.

Sonnad, J.R. and Goudar, C.T. (2007), "Explicit reformulation of the Colebrook-White equation for turbulent flow friction factor calculation", Industrial and Engineering Chemistry Research, Vol. 46 No. 8, pp. 2593-2600.

Winitzki, S. (2003). Uniform approximations for transcendental functions. In: Kumar, V., Gavrilova, M.L., Jeng, C., Tan, K., L'Ecuyer, P. (Eds.), Lecture Notes in Computer Science. Computational Science and Its Applications - ICCSA 2003, International Conference, Montreal, Canada, May 18-21, 2003 Proceedings, Part 1, 2667. Springer, Berlin/Heidelberg, pp. $780-789$. 


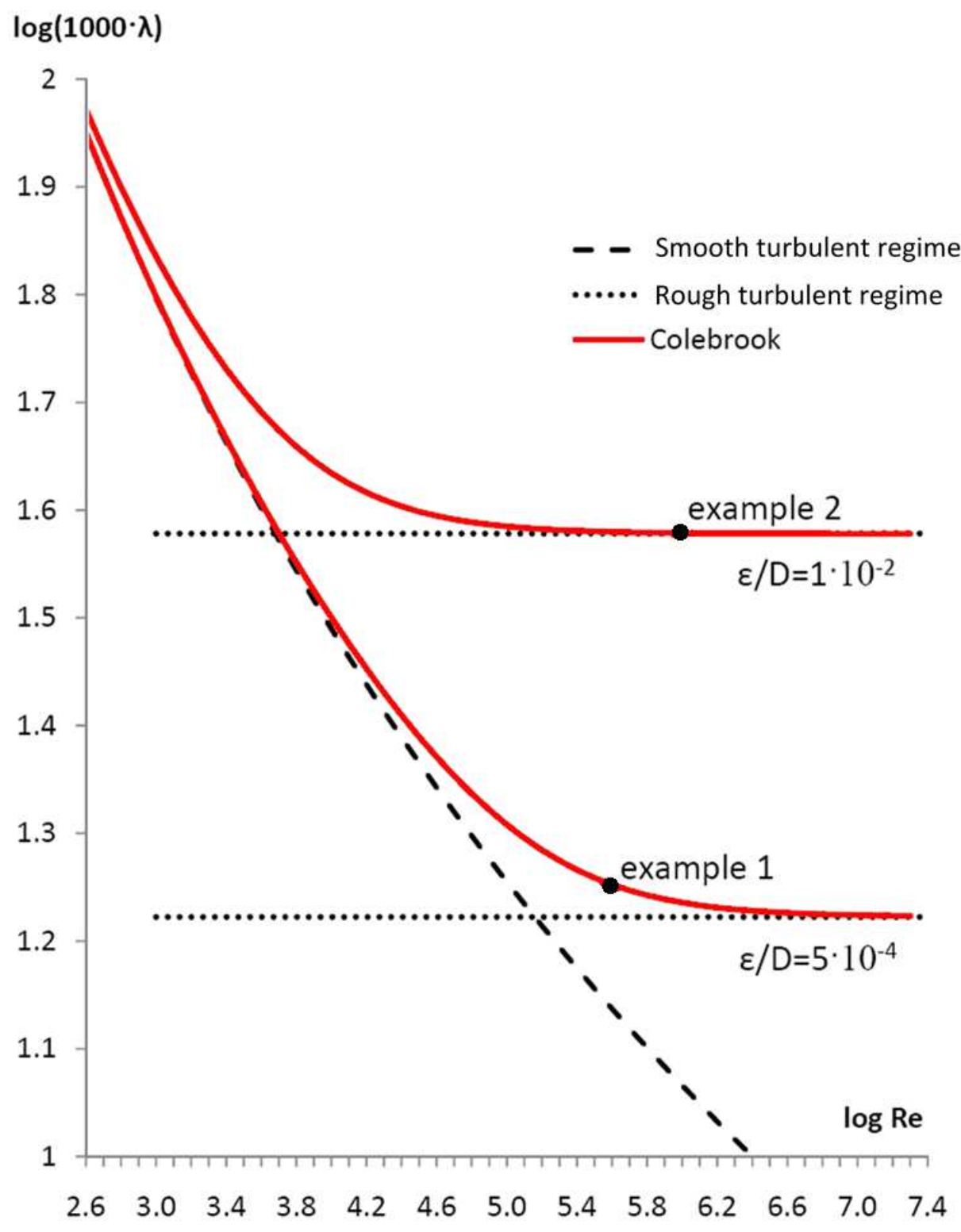

Figure 1. Colebrook relation make transitional curve between hydraulically smooth regime and turbulent rough regime $65 \times 82 \mathrm{~mm}(300 \times 300 \mathrm{DPI})$ 


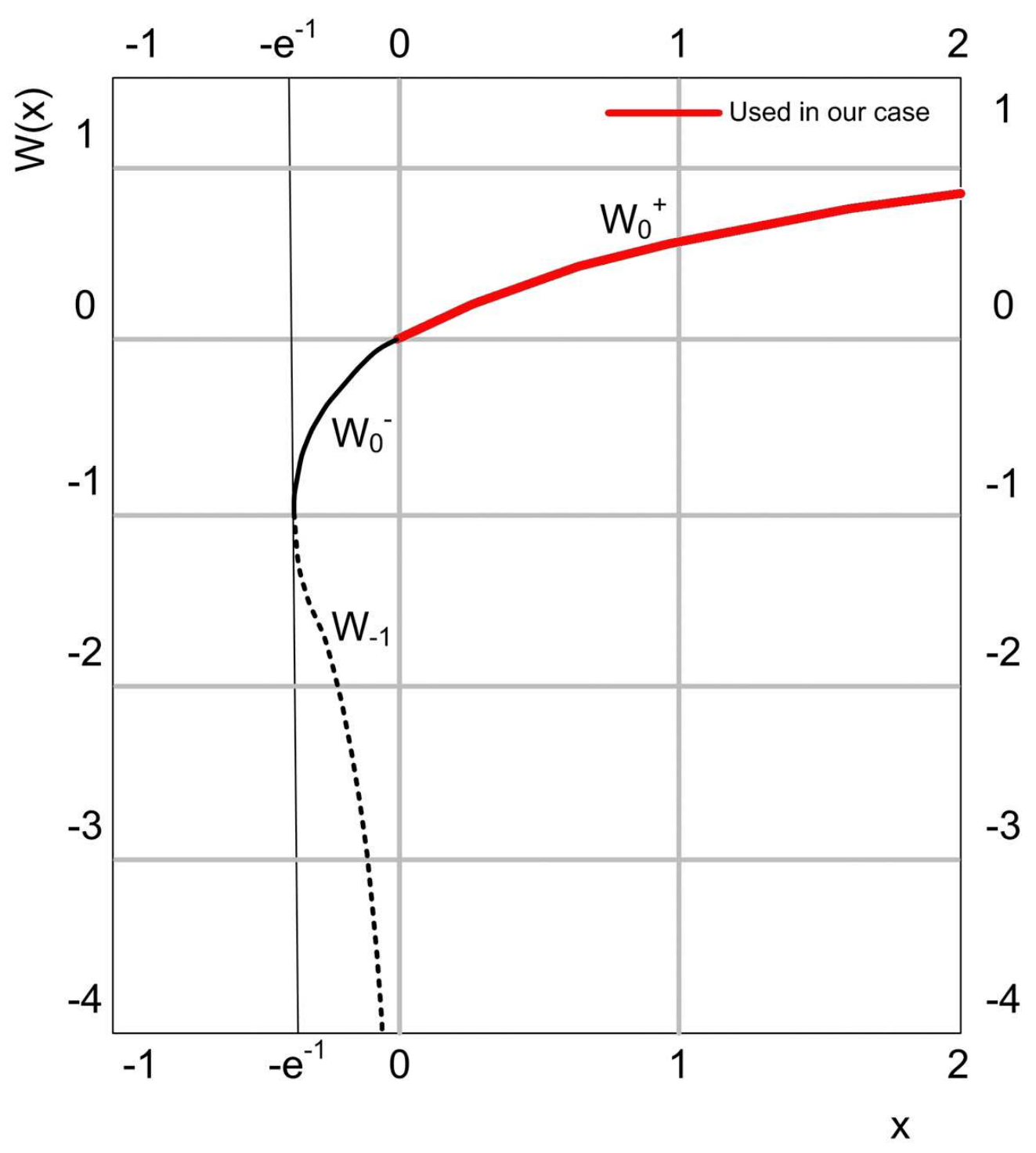

Figure 2. Detailed view of the Lambert W-function $99 \times 111 \mathrm{~mm}(300 \times 300 \mathrm{DPI})$ 


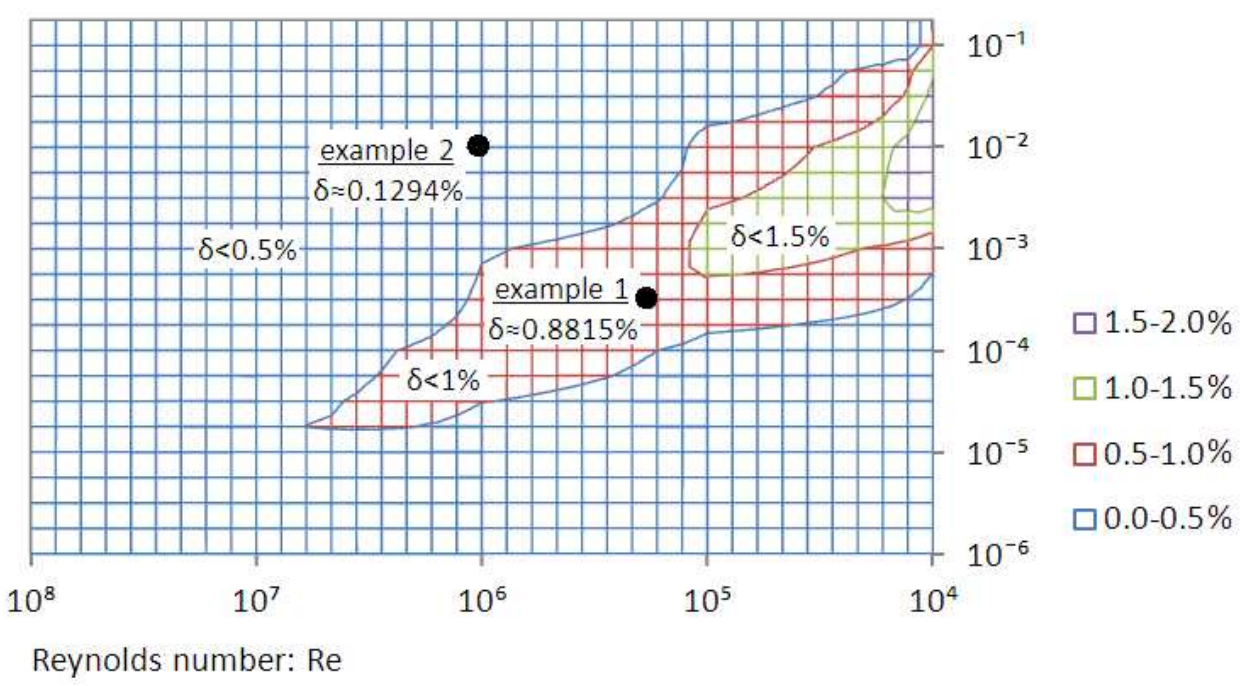

Figure 3. Distribution of error of solution of explicit Lambert W-function based Colebrook equation (5) by Boyd (1998) $65 \times 37 \mathrm{~mm}(300 \times 300$ DPI $)$ 


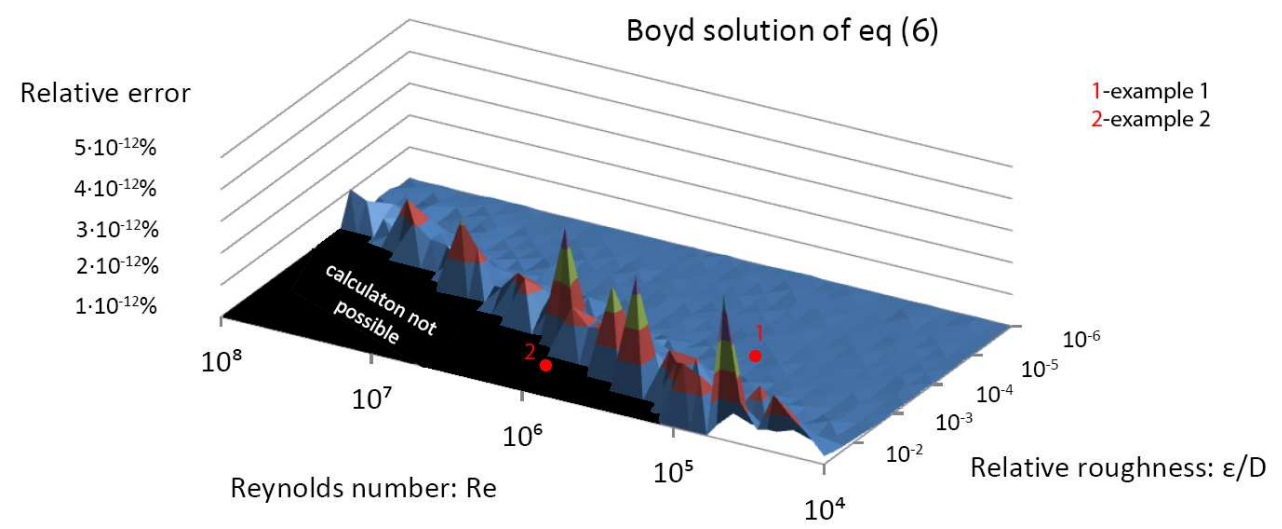

Figure 4. Distribution of error of solution of explicit Lambert W-function based Colebrook equation (6) by Boyd (1998)

$118 \times 50 \mathrm{~mm}(300 \times 300$ DPI $)$ 


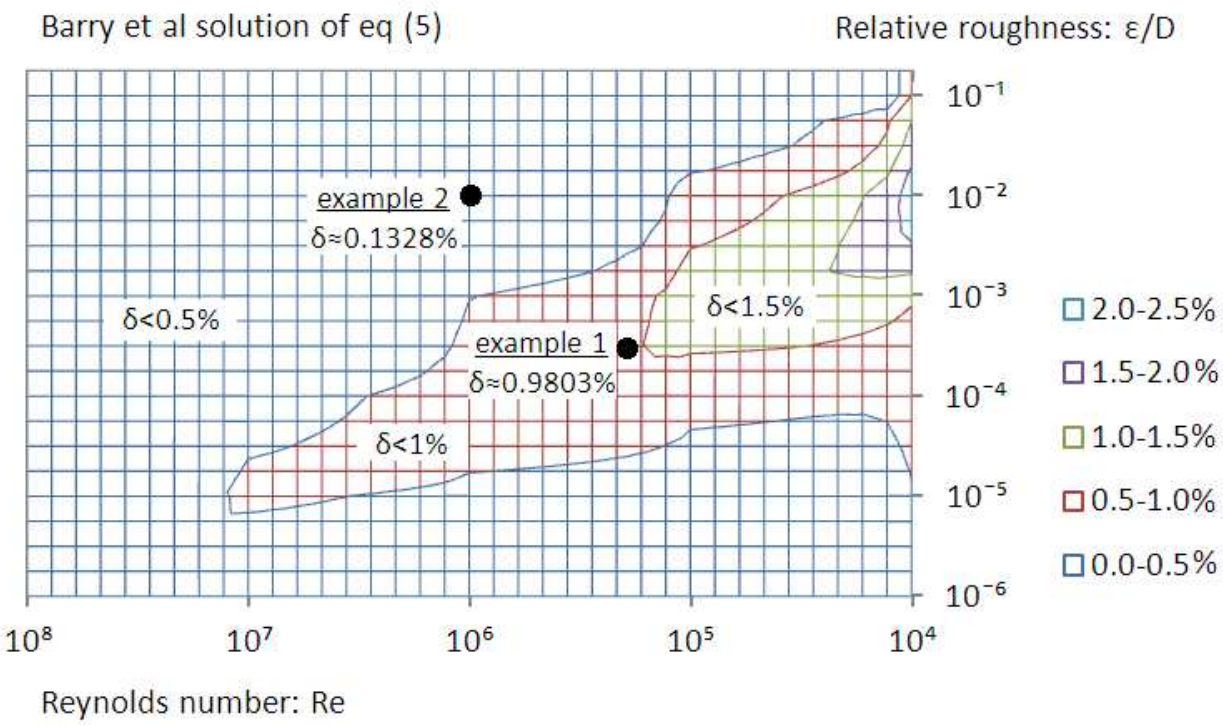

Figure 5. Distribution of error of solution of explicit Lambert W-function based Colebrook equation (5) by Barry et al (2000) $66 \times 38 \mathrm{~mm}(300 \times 300 \mathrm{DPI})$ 


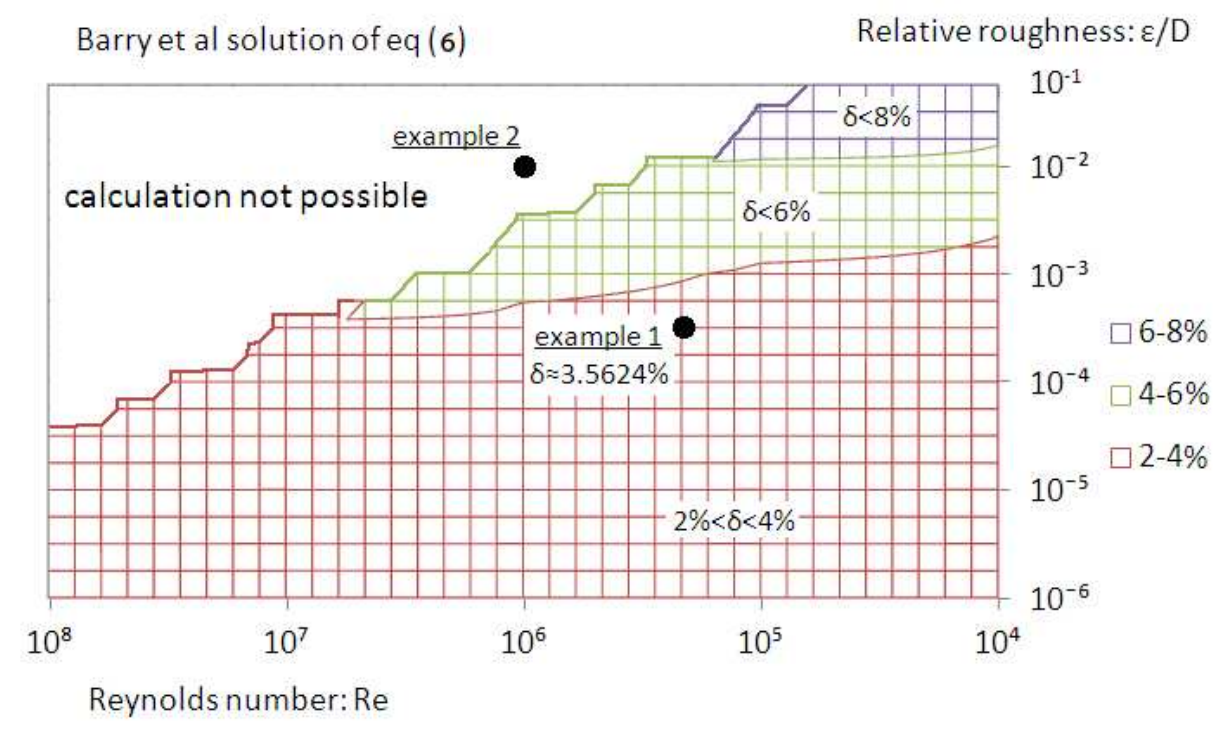

Figure 6. Distribution of error of solution of explicit Lambert W-function based Colebrook equation (6) by Barry et al (2000)

$63 \times 36 \mathrm{~mm}(300 \times 300 \mathrm{DPI})$ 


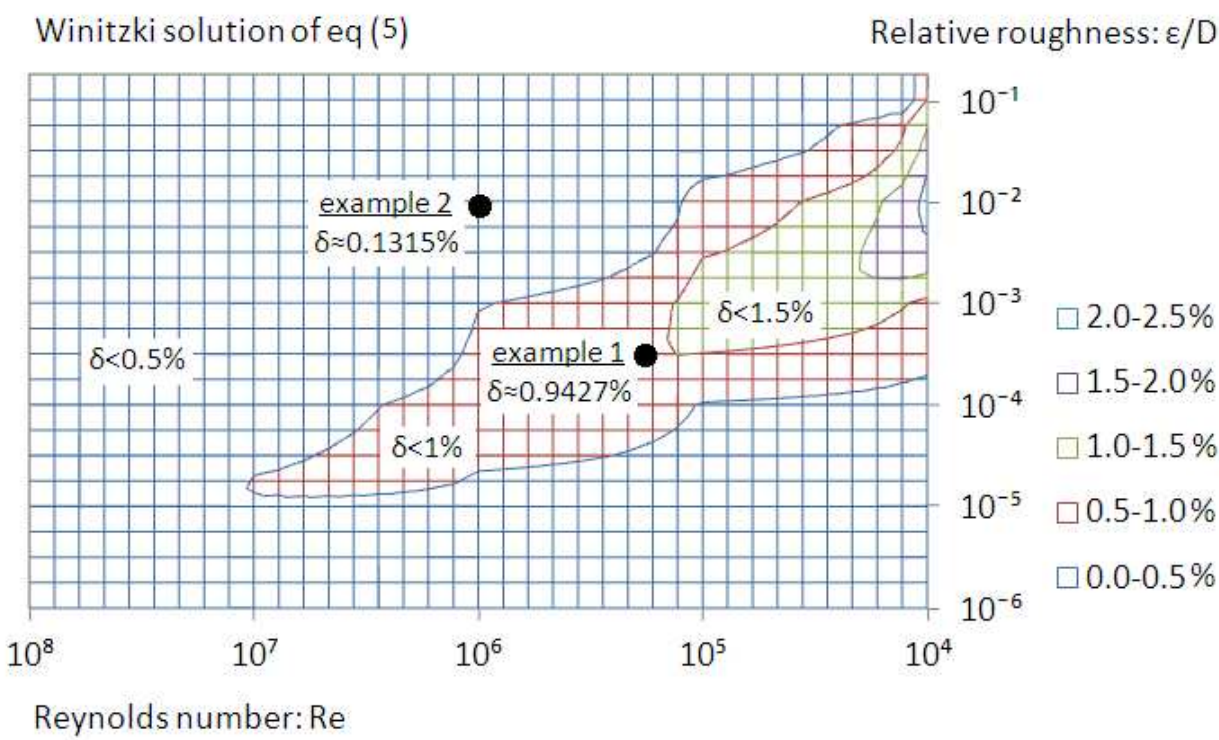

Figure 7. Distribution of error of solution of explicit Lambert W-function based Colebrook equation (5) by Winitzki (2003) $61 \times 36 \mathrm{~mm}(300 \times 300 \mathrm{DPI})$ 


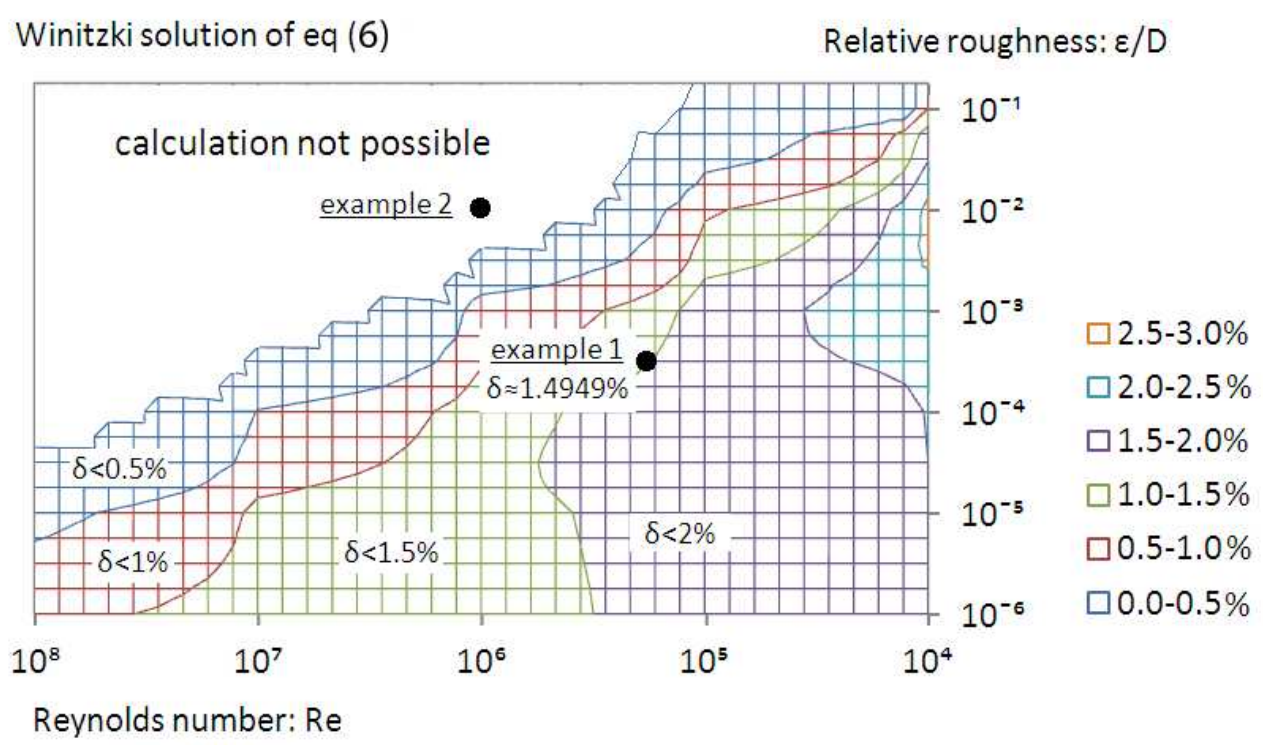

Figure 8. Distribution of error of solution of explicit Lambert W-function based Colebrook equation (6) by Winitzki (2003) $69 \times 40 \mathrm{~mm}(300 \times 300 \mathrm{DPI})$ 


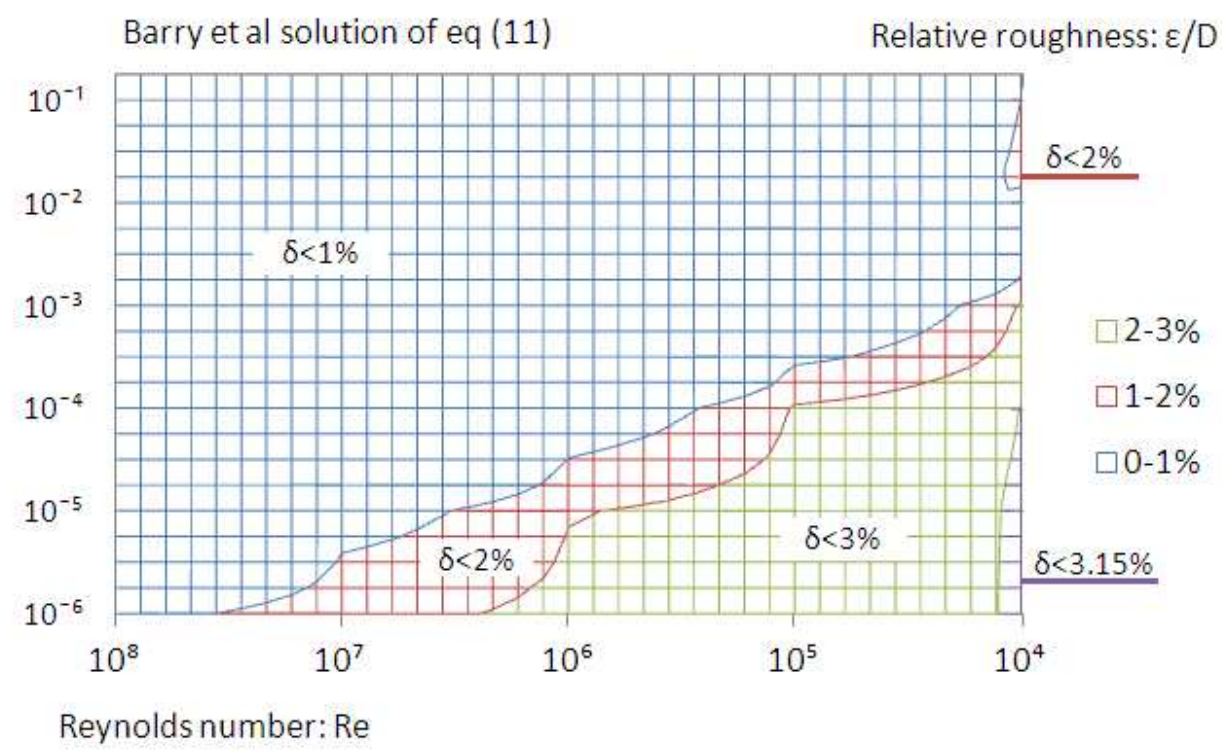

Figure 9. Distribution of error of solution of explicit Lambert W-function based Colebrook equation (11) by Barry et al (2000) $56 \times 33 \mathrm{~mm}(300 \times 300 \mathrm{DPI})$ 
Relative roughness: $\varepsilon / D$

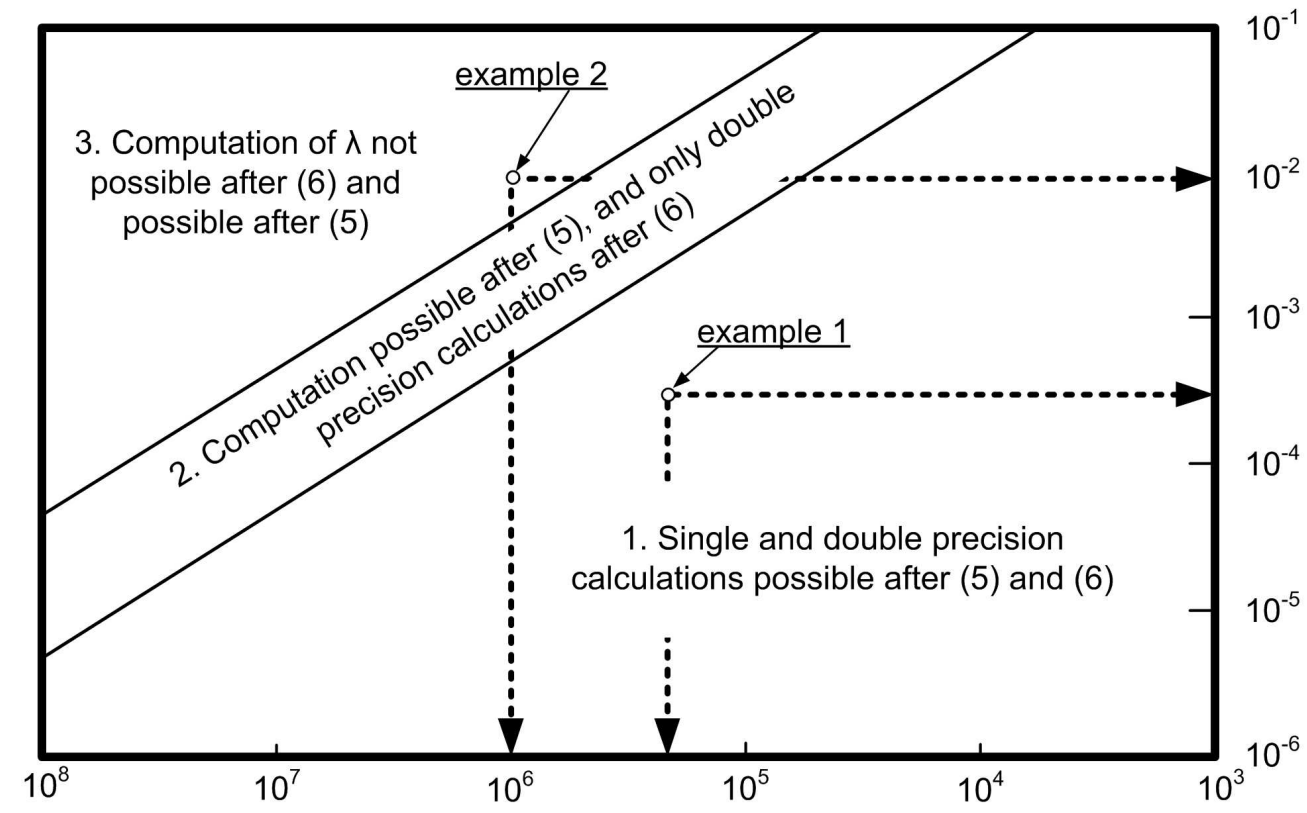

Reynolds number: Re

Figure 10. Constraints for using of a possible Lambert W function-based explicit Colebrook equation $166 \times 118 \mathrm{~mm}(300 \times 300 \mathrm{DPI})$ 
Table 1. Solutions of the explicit, Lambert W-function based Colebrook equation (5)

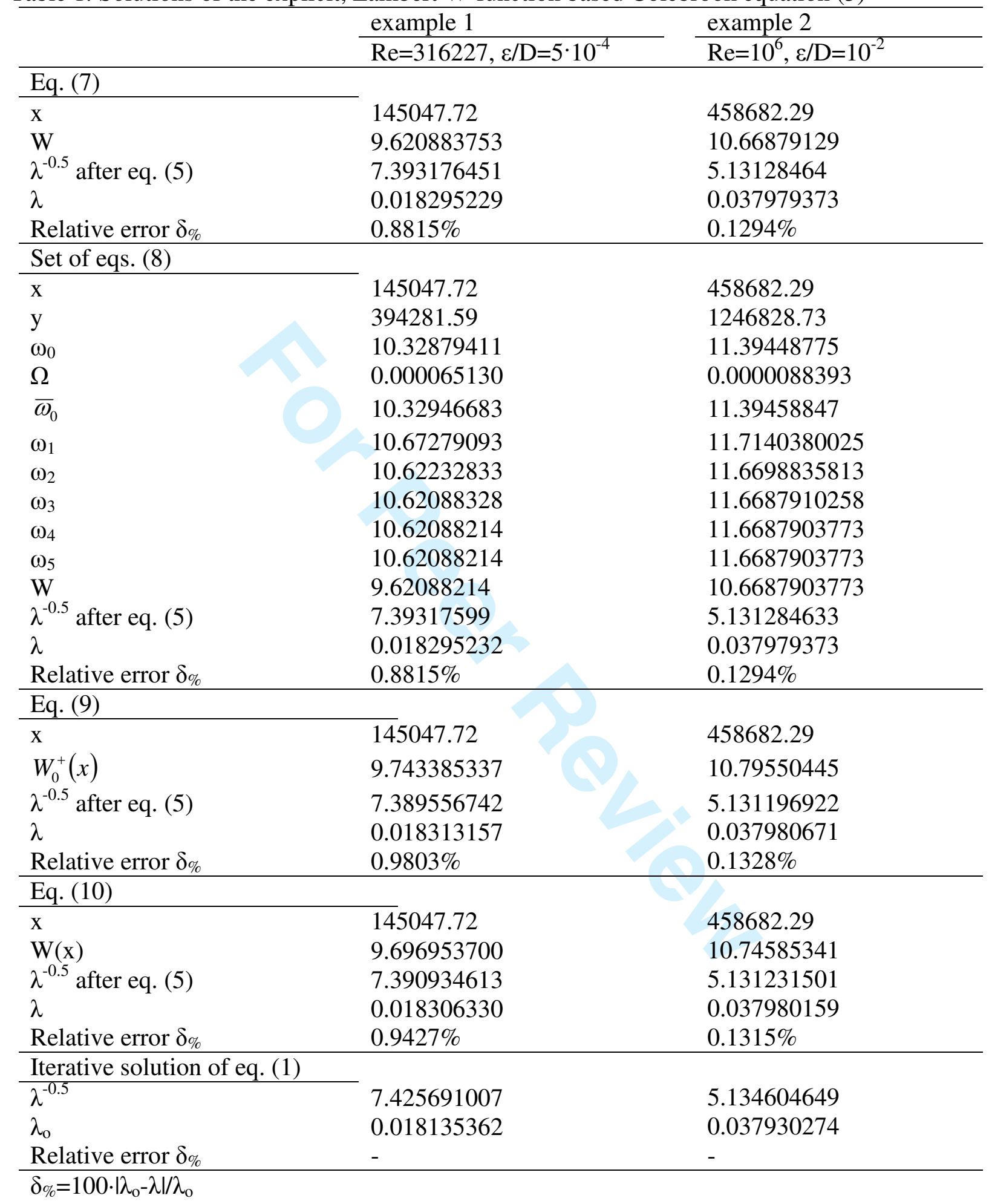


Table 2. Solutions of the explicit, Lambert W-function based Colebrook equation (6)

\begin{tabular}{|c|c|c|}
\hline & $\begin{array}{l}\text { example } 1 \\
\operatorname{Re}=316227, \varepsilon / D=5 \cdot 10^{-4}\end{array}$ & $\begin{array}{l}\text { example } 2 \\
\mathrm{Re}=10^{6}, \varepsilon / \mathrm{D}=10^{-2}\end{array}$ \\
\hline \multicolumn{3}{|l|}{ Eq. (7) } \\
\hline $\begin{array}{l}\mathrm{x}_{1} \\
\mathrm{~W} \\
\lambda^{-0.5} \text { after eq. }(6) \\
\lambda \\
\text { Relative error } \delta \%\end{array}$ & $\begin{array}{l}44791026258619.00 \\
28.09735354 \\
7.425691011 \\
0.018135363 \\
6.39 \cdot 10^{-8} \% \\
\end{array}$ & $\begin{array}{l}\text { Computer calculation not } \\
\text { possible }\end{array}$ \\
\hline \multicolumn{2}{|l|}{ Set of eqs. (8) } & \multirow[b]{2}{*}{$\begin{array}{l}\text { Computer calculation not } \\
\text { possible }\end{array}$} \\
\hline $\begin{array}{l}\mathrm{x} 1 \\
\mathrm{y} \\
\omega_{0} \\
\Omega \\
\bar{\omega}_{0} \\
\omega_{1} \\
\omega_{2} \\
\omega_{3} \\
\omega_{4} \\
\omega_{5} \\
\mathrm{~W} \\
\lambda^{-0.5} \text { after eq. }(6) \\
\lambda \\
\text { Relative error } \delta \% \\
\end{array}$ & $\begin{array}{l}44791026258619.00 \\
121754632756837.00 \\
28.95385161 \\
1.3882775 \cdot 10^{-31} \\
28.95385161 \\
29.1085521825 \\
29.0974181464 \\
29.0973535390 \\
29.0973535368 \\
29.0973535368 \\
28.0973535368 \\
7.42569100698 \\
0.01813536298 \\
4.63 \cdot 10^{-8} \%\end{array}$ & \\
\hline \multicolumn{2}{|l|}{ Eq. (9) } & \multirow[b]{2}{*}{$\begin{array}{l}\text { Computer calculation not } \\
\text { possible }\end{array}$} \\
\hline $\begin{array}{l}\mathrm{x}_{1} \\
W_{0}^{+}(x) \\
\lambda^{-0.5} \text { after eq. (6) } \\
\lambda \\
\text { Relative error }\end{array}$ & $\begin{array}{l}44791026258619.00 \\
28.253824116 \\
7.5615996248 \\
0.0174893086 \\
3.5624 \%\end{array}$ & \\
\hline \multicolumn{2}{|l|}{ Eq. (10) } & \multirow[b]{2}{*}{$\begin{array}{l}\text { Computer calculation not } \\
\text { possible }\end{array}$} \\
\hline $\begin{array}{l}\mathrm{x}_{1} \\
\mathrm{~W}(\mathrm{x}) \\
\lambda^{-0.5} \text { after eq. }(6) \\
\lambda \\
\text { Relative error } \delta \%\end{array}$ & $\begin{array}{l}44791026258619.00 \\
28.161979769 \\
7.4818246387 \\
0.0178642566 \\
0.4949 \%\end{array}$ & \\
\hline
\end{tabular}

